Title:

Enforcement policies: Frequency of inspection is more important than the severity of punishment

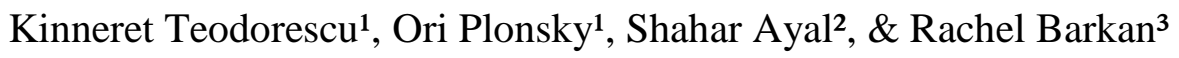 \\ 'Technion - Israel Institute of Technology \\ ${ }^{2}$ Interdisciplinary Center (IDC), Herzliya \\ ${ }^{3}$ Ben-Gurion University of the Negev
}

\begin{abstract}
:
External enforcement policies aimed to reduce violations differ on two key components: the probability of inspection and the severity of punishments. Different lines of research offer competing predictions regarding the relative importance of each component. In three incentive compatible studies, students and Prolific crowdsourcing participants $\left(\mathrm{N}_{\text {total }}=430\right)$ repeatedly faced temptations to commit violations under two enforcement policies. Controlling for expected value, the results indicated that a policy combining High probability of Inspection with Low Severity of fine (HILS) was more effective than a policy combining Low probability of Inspection with High Severity of fine (LIHS). Consistent with the prediction of Decisions from Experience research, this finding held even when the severity of the fine was stated in advance to boost deterrence. In addition, the advantage of HILS over LIHS was greater as participants' baseline rate of violation (without enforcement) was higher, implying that HILS is more effective among frequent offenders.
\end{abstract}

Keywords: Behavioral ethics, enforcement, decisions from experience, behavioral economics, policy making, cheating, learning 
Enforcement Policies: Inspection Frequency vs. Punishment Severity

\section{Enforcement policies: Frequency of inspection is more important than the severity of punishment}

Texting while driving, jaywalking, littering, not wearing facemasks and neglecting to keep social distancing during the Covid-19 pandemic are but a few examples of seemingly negligible violations that accumulate fast, with potentially dire social consequences. The prevalence of these violations highlights the shortcomings of reasoning with, or appealing to, people's civic duty, and point to the need for regulation and enforcement. External enforcement is composed of the probability of inspection and the severity of the punishment delivered upon detection (Becker, 1968). Clearly, the combination of complete monitoring and severe punishments is the fastest way to shape behavior. However, due to the limited resources devolved to monitoring, and the negative consequences of severe punishment (e.g., reactance), policy makers usually resort to two main compensatory strategies. One solution opts for less frequent inspection but greater severity of punishment and counts on its potential for deterrence. The other solution opts for close monitoring, and assumes that when the inspection rate is high, minimal or even symbolic punishment will suffice. The current paper examined the effectiveness of these two enforcement strategies in reducing violations.

Several theoretical perspectives are relevant here. The economic perspective views people as rational agents, whose actions are governed by cost-benefit analysis. Accordingly, people will commit violations if their expected utility (accounting for potential gains and losses) is positive (Becker, 1968). Under the additional assumption that most people are not risk-seeking, Becker's model predicts that people will be more sensitive to the severity of punishment than to the probability of detection. Several studies in experimental economics support this notion (e.g., Anderson \& Stafford, 2003; Block \& Gerety, 1995; Engel \& Nagin, 2015; Friesen, 2012). The economic perspective therefore implies that delivering severe punishments, even rarely, is the most effective enforcement strategy.

A psychological perspective pits the external gain associated with violations against the internal cost of damage to one's self-perception (e.g., Shalvi et al., 2011; Abeler et al., 2014). Self-maintenance theory (Mazal et al., 2008) suggests that aiming to perceive oneself as highly moral creates a barrier to acts of violation. Specific mechanisms allow people to engage in some wrongdoing but at the same time keep their sense of morality intact (Ayal \& Gino, 2011; Barkan et al., 2015). Supporting this notion, findings in behavioral ethics indicate that although participants commit violations, they do so to a limited extent rather than to the maximum possible (e.g., Gino et al., 2009, 2013; Gneezy et al., 2013; Mazar et al., 2008). This self-curbing tendency 
has frequently been documented in common experimental tasks that eliminate detection and fines (Gerlach et al., 2019). Certain findings show that violation rates are generally insensitive to external settings, and remain relatively stable regardless of significant changes in the potential profit (e.g., Fischbacher, \& Föllmi-Heusi, 2013) or in the chances of being caught (Mazar et al., 2008). If people are indifferent to external settings as implied by self-maintenance theory, the effectiveness of either enforcement strategy should be similar and limited.

Findings in the field of behavioral ethics typically rely on a single, or a short sequence of choices (Gerlach et al., 2019) and generally do not address the long-term effects of external enforcement. Extending experimental tasks to repeated settings, introduces learning as a third perspective to consider. Research on repeated decisions from experience indicates that choice behavior is more sensitive to the frequency of experienced outcomes than to their magnitude (e.g., Teodorescu \& Erev, $2014 \mathrm{a} \& \mathrm{~b})$. This line of research shows that when people learn from experience, they tend to behave as though they underweight small probability outcomes (e.g. Barkan et al., 1998; Hertwig et al., 2004; Camilleri \& Newell, 2011; Teodorescu et al., 2013; Plonsky \& Teodorescu, 2020). Altogether, these findings hint that infrequent large fines may not have the intended outcome, and suggest instead that frequent small fines should be more effective at decreasing violations.

This implies three competing hypotheses with respect to the effectiveness of the two enforcement strategies:

H1 - Economic perspective: Rare large fines should be more effective than frequent small fines in deterring violations.

$\mathrm{H} 2$ - Self maintenance theory: Both rare large fines and frequent small fines should have a similar and limited impact on the deterrence of violations.

H3 - Decisions from experience: Frequent small fines should be more effective than rare large fines at deterring violations.

In three studies, we tested these competing hypotheses by assessing which enforcement strategy is more effective in settings where people can repeatedly engage in small violations. We utilized the dots task (e.g., Gino et al., 2010; Hochman et al., 2016), a perceptual task that creates a conflict between accuracy and profit maximization (when incorrect responses yield higher payoffs than correct responses). This task has three advantages. It consists of many trials, thus permitting the examination of violation behavior over time. It allows for incorporation and systematic manipulation of the probability of inspection and the severity of fines, while also 
controlling for the expected value (EV) of violations. Finally, it constitutes a simplistic simulation of real-life settings where people know enforcement is possible, and can learn the likelihood of being caught from experience (Harel \& Segal, 1999).

\section{Pilot study}

The pilot study tested the three competing hypotheses by comparing violation rates under two partial enforcement conditions: rare large fines vs. frequent low fines. In both conditions, the EV of violations was identical. Two control conditions administered either no external enforcement or full external enforcement.

\section{Method}

Participants. Forty-two undergraduate students from a major university participated in a 1-hour lab study. Payment was contingent on performance $(M=46.0$ NIS, $S D=4.0)$. Each participant played 768 repeated trials, half of which contained a monetary temptation to commit a violation (i.e., overall, we analyzed 32,256 observations).

Basic task. We utilized the dots task (Gino et al., 2010; Hochman et al., 2016) and presented 20 dots distributed unevenly between two adjacent rectangles. On each trial participants were asked to decide whether there are more dots on the right or left rectangle. One side always yielded 1 point (0.01 NIS) whereas selecting the other side always yielded 10 points ( $0.1 \mathrm{NIS})$, irrespective of the correct answer. Thus, the payoff rule motivated 'accuracy-violations' such that participants could profit by selecting the more rewarding side on tempting trials where the correct answer is the less rewarding side. The more rewarding side was counterbalanced across participants, and remained the same for each participant throughout the experiment. The experiment consisted of four "games" of 192 trials each. In each game, half of the trials presented more dots on the more rewarding side (non-tempting trials), and half presented more dots on the less rewarding side (tempting trials). Participants played the task continuously. The order of trials in each game was random. Each trial started with a $0.5 \mathrm{sec}$ presentation of a fixation point, followed by a $1 \mathrm{sec}$ dots screen. Next, participants were asked to indicate which side had more dots by clicking on one of two boxes on the screen. Last, a $1 \mathrm{sec}$. feedback screen presented the trial's payoff.

On top of this basic incentive structure, we modified the dots task to include conditions with and without inspection of the correctness of the answer. Without inspection, the feedback screen only presented the trial payoff. To signal inspection, the feedback screen turned red and if 
an incorrect answer was detected, a fine appeared on the screen and was subtracted from the trial's payoff. Figure 1 depicts two trials on the modified dots task: one without inspection and one with inspection.

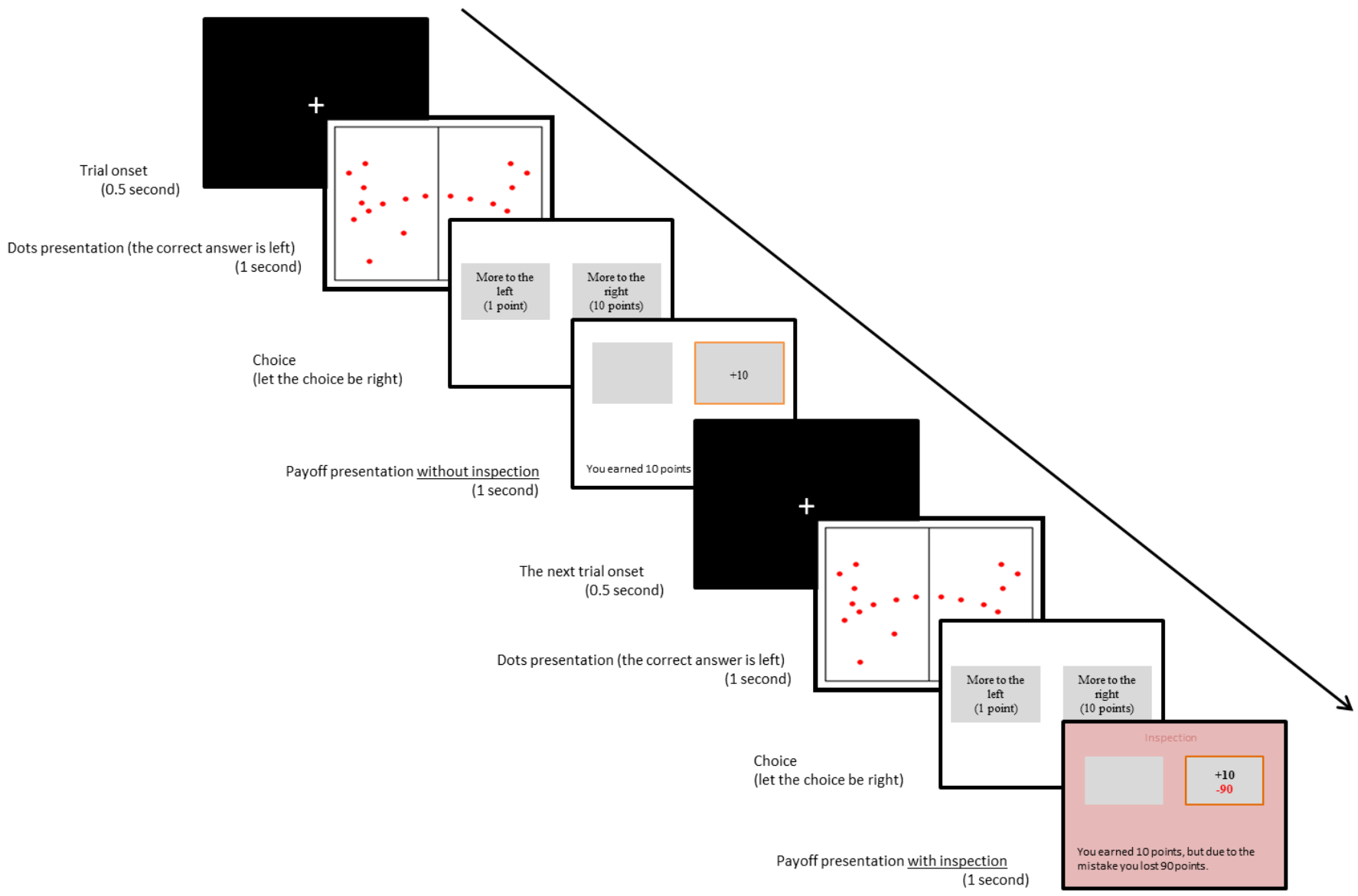

Figure 1. Timeline example of two trials: the first trial without inspection and the second with inspection under an enforcement policy with severe punishment (fine $=-90$ points).

Experimental design. The games tested the four enforcement conditions which differed in the rate of inspection and the severity of the punishment. The No-Enforcement (NE) condition corresponded to the original cheating condition on the dots task without inspection and fines. In the Full-Enforcement (FE) condition, each trial was inspected and each incorrect answer was fined -18 points. In the two partial enforcement conditions the inspection rate and punishment severity varied. In the High-Inspection-Low-Severity (HILS) condition, 90\% of the trials were inspected and each detected violation was fined -10 points. In the Low-Inspection-High-Severity (LIHS) condition, only $10 \%$ of the trials were inspected, but each detected violation was fined -90 points. Therefore, the EV for an accuracy violation was identical in the two partial enforcement conditions. In addition, within each of the two partial enforcement conditions, the EV of an 
accuracy violation was identical to the fixed payoff that resulted from reporting the correct answer. Table 1 summarizes the experimental conditions. Utilizing a complete within-subject design participants engaged in all the conditions (i.e., 4 "games" presented in random order).

Table 1: Experimental conditions in the pilot study (EV=Expected Value).

\begin{tabular}{|c|c|c|c|}
\hline Condition & P(inspection) & Fine & $\begin{array}{c}\text { EV (incorrect)* } \\
\text { when reporting the incorrect } \\
\text { answer is tempting }\end{array}$ \\
\hline $\begin{array}{c}\text { No Enforcement } \\
\text { (NE) }\end{array}$ & 0 & - & 10 \\
\hline $\begin{array}{c}\text { Low Inspection } \\
\text { High Severity } \\
\text { (LIHS) }\end{array}$ & 0.1 & 90 & 1 \\
\hline $\begin{array}{c}\text { High Inspection } \\
\text { Low Severity } \\
\text { (HILS) }\end{array}$ & 0.9 & 10 & 1 \\
\hline $\begin{array}{c}\text { Full Enforcement } \\
\text { (FE) }\end{array}$ & 1 & 18 & -8 \\
\hline
\end{tabular}

* Compared with $\mathrm{EV}($ correct $)=1$ for tempting trials in all conditions.

Procedure. Participants read the instructions, signed a consent form and engaged in the dots task. The participants were informed that inspection could occur, and that in case of inspection the feedback screen would turn red and that detection of an incorrect answer would be fined. However, we did not specify the enforcement policy; thus, participants had to infer the probability of inspection and the severity of punishment (i.e. the fine) based on their experience in each game. Participants were informed when one game was over and a new one began, and that in each game the possibility of inspection as well as the severity of the punishment could be different.

\section{Results}

The rate of accuracy violations was calculated as the difference between the proportions of 'beneficial errors' (i.e., selection of the incorrect but more rewarding side) and 'detrimental errors' (i.e., selection of the incorrect but less rewarding side) (Hochman et al., 2016). The mean rate of accuracy violations was the highest without enforcement (47.5\% in NE condition) and the 
lowest with full enforcement (3.0\% in FE condition). This suggests that accuracy violations were mostly intended, and favored profit over accuracy. Without enforcement, participants tended, on average, to violate accuracy for profit on just under half of the tempting trials. Note that only half of the trials were tempting. Thus, the accuracy-violation rate was below $25 \%$ and far below the maximum rate of $50 \%$, thus leaving room for moral justification, as suggested by selfmaintenance theory.

Not surprisingly, the two partial enforcement conditions were less effective in reducing violations than full enforcement. Importantly however, a clear advantage emerged for probability of inspection over severity of punishment (despite the control for expected values). Consistent with the decisions from experience hypothesis (H3), the rate of accuracy violations was low when the enforcement rule prioritized probability, and significantly higher when the enforcement rule prioritized severe punishment (8.53\% vs. $27.69 \%$ in the HILS and LIHS conditions respectively $t(41)=5.9, p<0.001)$.

\section{Main studies}

The main studies compared the two partial enforcement policies directly in a betweensubject design. Due to COVID-19 restrictions, these studies were run online ${ }^{1}$. To increase power and reduce noise, we increased the number of tempting trials. In each study, participants engaged in two games. They first completed the original dots task without enforcement and then repeated the task under either the HILS or LIHS enforcement conditions. This design served to (1) measure the effectiveness of each enforcement policy in reducing accuracy violations, and (2) categorize participants according to their baseline tendencies without enforcement. Several studies that have examined individual differences suggest that certain people react to incentives to lie, whereas others do not (e.g., Gneezy et al., 2013; Hilbig \& Zettler, 2015; Kajackaite \& Gneezy, 2017; Feldman, 2018). Establishing individuals' baseline thus made it possible to identify participants who rarely misreport, even without enforcement, and therefore examine the effectiveness of partial enforcement on those participants for whom enforcement mattered. The pre-registered hypotheses were: (1) HILS enforcement will reduce violations more than LIHS (in line with H3 and the pilot results) and (2), this effect will be most pronounced in participants with high baseline violation rates.

\footnotetext{
${ }^{1}$ The transition to online participants was not straightforward. We ran three online studies to calibrate the online paradigm. The results of these studies are detailed in the supplemental material.
} 
Enforcement Policies: Inspection Frequency vs. Punishment Severity

\section{Study 1}

\section{Method}

Participants. 202 participants were recruited via the crowdsourcing platform Prolific to the study, which lasted about 17 minutes. Participants received a show-up payment of $£ 1.45$ and a bonus based on the number of points they earned during the task $(M=£ 1, S D=0.29)$. The number of participants was determined a-priori according to a power analysis based on a preliminary study (available in the supplemental material). The pre-registration (available at https://aspredicted.org/blind.php?x=4ux6ja) states: "The effect size $d$ for the main effect between groups in a pilot experiment was 0.395. With this effect size, and for $85 \%$ power and alpha of 0.05 , we require at least 94 participants in each group, after exclusions. We will hence start with 100 participants in each group, exclude any participants as necessary, and will complement the sample size to 94 in each group if necessary." We pre-registered an exclusion criterion, omitting participants who made more than 35\% detrimental errors in the baseline no-enforcement game from the analysis. Six participants were excluded based on this criterion. The final sample was composed of 196 participants (104 in HILS, 94 in LIHS).

Experimental Task. We employed the dots task from the pilot study, with several changes aimed to increase power and make accuracy violations at baseline more tempting so as to avoid a floor effect. First, we replaced the vertical line with a diagonal, and always presented 17 vs. 13 dots on each side on each trial (the location of the dots within each triangle was random, thus naturally creating varied difficulty levels but with reduced variance). Second, we increased the temptation for accuracy violations slightly: the payoff still rewarded a response to one side regardless of accuracy (10 points), but we omitted the reward for the alternative response ( 0 points). Third, to obtain more data points, each game now had 120 trials, out of which 80 trials were tempting (the incorrect answer was rewarding) and 40 trials were non-tempting (the correct answer was rewarding). Last, we adjusted the inspection to include green and red screens rather than only red. Specifically, under inspection, if the answer was incorrect, the feedback screen turned red (and a fine was subtracted from the trial payoff), but if the answer was correct, the inspection screen turned green. This differed from the pilot study where all inspected trials turned red, for both the correct and incorrect answers.

Experimental design. All participants started with a "No-Enforcement" (NE) game and were then randomly assigned to either the HILS or the LIHS condition. The High Inspection Low Severity (HILS) condition had a .90 probability of inspection and a fine of -11 points. The Low Inspection High Severity (LIHS) condition had a .10 probability of inspection and a fine of -99 points. We adjusted the fines to keep the Expected Value (EV) of accuracy violation on the 
tempting trials identical in the two conditions (i.e., $\mathrm{EV}=0.1$, which is slightly larger than the zero points obtained from reporting the correct answer).

Procedure. Participants signed a consent form, read the instructions and then played two games. All the participants started with a "No-Enforcement" (NE) game. Next, the possibility of enforcement was explicitly introduced and participants played a second game according to the experimental condition. As in the pilot study, we informed participants that inspection is possible but did not specify the probability of inspection or the amount of the fine, which they had to infer through experience. Upon completion, we asked participants to guess the goal of the study, and whether they would have acted differently if they had to do the task again. Participants were then notified about their bonus payment and received their task-completion code.

Data analysis. As in the pilot study, accuracy-violation rates were calculated as the difference between 'beneficial errors' (percentage of incorrect answers in tempting trials out of all tempting trials) and 'detrimental errors' (percentage of incorrect answers in non-tempting trials out of all non-tempting trials). Given the within-subject aspect of the experimental design, the dependent variable for the main analysis was the difference between accuracy-violation rates in the enforcement and baseline games where a larger difference indicated the greater effectiveness of enforcement (we expected both enforcement conditions to reduce accuracy violations as compared to their respective baselines). Based on the results of the pilot, and consistent with the decisions from experience hypothesis (H3), we expected a larger difference in the HILS enforcement condition and a smaller difference in the LIHS enforcement. Hence, we preregistered a one-sided Welch t-test to examine this main hypothesis and noted that if the ShapiroWilk test will have indicated a violation of the assumption of normality, we will use the Mann Whitney U test instead.

A secondary analysis further investigated the expected effect of enforcement on different types of participants. Using the accuracy-violation baseline rates in the first game (NE), three types of participants were preregistered. Participants whose baseline accuracy-violation rate was in the lowest quartile (Q1) were labelled Low Accuracy-violators. Participants whose baseline accuracy-violation rate was in the highest quartile (Q4) were labelled High Accuracy-violators. Participants whose baseline accuracy-violation rate fell in the interquartile range (Q2 \& Q3) were labelled Medium Accuracy-violators. We calculated quartiles across all participants (since the baseline game was identical). Consistent with the pre-registration, we regressed the accuracyviolation rates in the second game on the enforcement condition (HILS/ LIHS), the type of participant (Low/Med/High Accuracy-violators), and their interaction. 
Enforcement Policies: Inspection Frequency vs. Punishment Severity

\section{Results}

Testing the main hypothesis. Figure 2a presents the main finding. On average, accuracyviolation rates decreased by $23.3 \%$ under the HILS enforcement that prioritized probability $(S D=$ 25.7; from $33.6 \%$ in the No-Enforcement game to $12.2 \%$ in the with-enforcement game). In contrast, accuracy-violation rates decreased by only $10.3 \%$ under the LIHS enforcement that prioritized punishment severity $(S D=21.1$; from $32.6 \%$ in the No-Enforcement game to $22.4 \%$ ). Because a Shapiro-Wilk test rejected the assumption of normality for the accuracy-violation rates $(p<.001)$, and consistent with our pre-registration plan, we used a Mann-Whitney U-test to test our hypothesis. Supporting our hypothesis (H3), the test indicated that compared to baseline the reduction in accuracy-violation rates in the HILS condition ( $\operatorname{mdn}=15 \%$, range $[-18,133])$ was greater than in the LIHS condition ( $\mathrm{mdn}=7.5 \%$, range $[-25,90]), \mathrm{U}=6304, p<.001$, one-tailed test, effect size $r=0.25$.

One possible concern was that the inspection screens could have confounded the experience of enforcement with simple accuracy feedback. That is, frequent inspection (HILS) could have improved participants' perceptual ability, which in turn could have led to fewer accuracy violations in both the tempting and non-tempting trials. To test for this possibility, we examined participants' accuracy in the non-tempting trials. If the feedback improvement explanation holds, there should be a greater improvement in accuracy between the first and the second block in the HILS condition compared to the LIHS condition. There was no evidence for this. Surprisingly, a Welch two sample t-test for the non-tempting trials revealed a significant difference between the two groups but in the opposite direction $(M=0.9 \%, S D=6.0$ vs. $M=$ $-2.4 \%, S D=6.0$ in the LIHS and HILS conditions respectively), $t(173.5)=-2.87, p=.005$. These results rule out the alternative explanation of simple feedback improvement. 


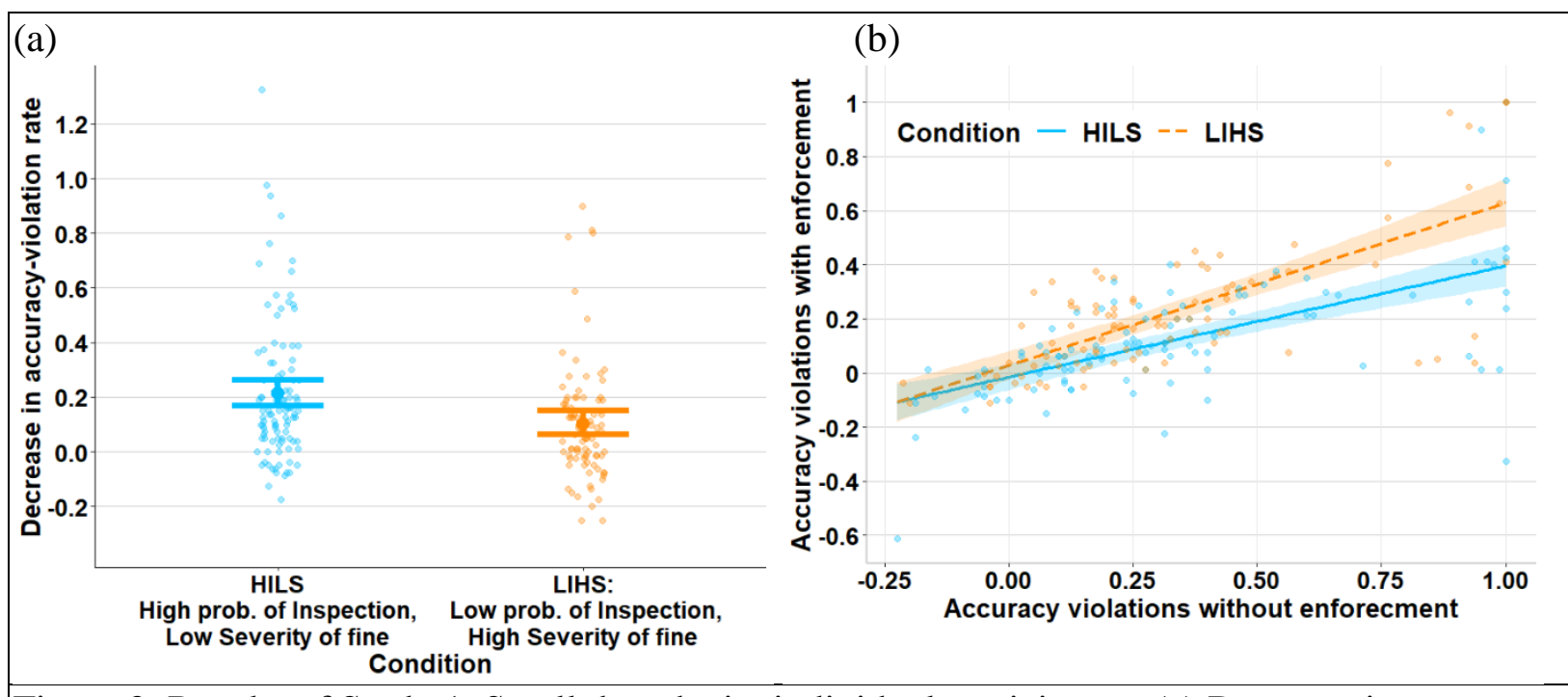

Figure 2. Results of Study 1. Small dots depict individual participants. (a) Decrease in accuracyviolation rates from baseline to the enforcement block by condition. Error bars represent $95 \%$ bootstrapped CI for the mean. (b) Regression lines of the accuracy-violation rates in the enforcement blocks on the accuracy-violation rates in the No-Enforcement block, by condition. Shading around the lines are the $95 \%$ CIs.

Testing the secondary hypothesis. As pre-registered, we categorized participants into three types according to their baseline accuracy-violation rates in the No-Enforcement baseline game. Low Accuracy-violators (Q1) were those with accuracy-violation rates below $12.5 \%$ at baseline, High Accuracy-violators (Q4) had accuracy-violation rates above $43.8 \%$ at baseline, and Medium Accuracy-violators (Q2 \& Q3) were those with accuracy-violation rates in between (12.5-43.8\%). The three baseline types were consistently reflected in the second enforcement game. Specifically, the Low Accuracy-violators and the High Accuracy-violators consistently exhibited the lowest and the highest accuracy-violation rates respectively in the second game with enforcement. Importantly, all three types consistently manifested lower accuracy-violation rates when enforcement prioritized probability (HILS) compared to the accuracy-violation rates when enforcement prioritized severity of punishment (LIHS). The regression analysis indicated a main effect for condition, $F(1,192)=13.8, p<.001$, as well as a main effect for type, $F(2,192)=59.8$, $p<.001$. However, the interaction was not significant $F(2,192)=0.94, p=.39$; thus this analysis failed to find significant evidence for the hypothesis that High Accuracy Violators were more likely to be influenced by the enforcement condition.

The lack of evidence for the secondary hypothesis might reflect the loss of power involved with the categorization that reduced the continuous variable of accuracy-violation rates to a 3level variable. To test this possibility, we conducted a follow-up (unregistered) analysis repeating the regression above, but replacing the 3-level type with the individual accuracy-violation rate 
scores in the No-Enforcement game. This analysis yielded a significant interaction $F(1,194)=$ $5.79, p=0.017, b=0.19,95 \% \mathrm{CI}[0.03,0.35]$, partial $f^{2}=0.03$. The fitted regression lines for the two conditions (Figure $2 b$ ) showed that the difference between the two enforcement conditions was more pronounced when the accuracy-violation rates in the No-Enforcement game were higher. Specifically, the simple slopes for the accuracy-violation rates with enforcement on the accuracy-violation rates without enforcement were $0.41,95 \%$ CI [0.31, 0.51] for HILS and 0.60, $95 \%$ CI [0.48, 0.72] for LIHS.

\section{Study 2}

In many real-life situations, the severity of sanctions is known ahead of time. That is, although the probability of inspection is unknown (Harel \& Segal, 1999), the magnitude of the fine is sometimes public knowledge (e.g., via traditional or social media platforms). Accordingly, arguments in favor of severe sanctions are rather common and usually emphasize the deterrence effect. Moreover, studies in behavioral economics suggest that the presentation of small sanctions might constitute too low a price which could legitimize violations (e.g., Gneezy \& Rustichini, 2000). Thus, knowing the severity of the punishment in advance might undermine the effectiveness of enforcement that prioritizes probability. Nevertheless, findings from the literature on decisions from experience suggest that repeated on-going experience with feedback quickly eliminates the initial effects of descriptive information (e.g., Jessup et al., 2008; Teodorescu et al., 2013; Marchiori et al., 2015; Erev et al., 2017). We therefore hypothesized that the observed advantage of prioritizing probability (HILS) over severity of punishment (LIHS) would hold even with a-priori information about the magnitude of the fine.

\section{Method}

Participants. 196 participants who did not participate in the previous study were recruited via the crowdsourcing platform Prolific to complete this follow-up study. They were paid $£ 1.45$ for participating and were given a bonus contingent on their accumulative points gained during the experiment $(M=£ 1.02, S D=0.29)$. Four participants were excluded based on the exclusion criterion of $35 \%$ 'detrimental errors' or more, resulting in 94 vs. 98 participants in the HILS and LIHS conditions respectively.

Task and procedure. We used the same task, design, and procedure with one exception: the addition of information about the size of the fine (the probability of inspection remained unspecified). Participants now expected fines of -11 or -99 points in the HILS and LIHS conditions respectively. 
Enforcement Policies: Inspection Frequency vs. Punishment Severity

\section{Results}

Testing the main hypothesis. The findings replicated the results of Study 1, although the effects were smaller. Figure 3 a presents the main result. In the HILS condition, the mean accuracy-violation rate decreased by $21.8 \%$ ( $S D=28.6$; from $35.4 \%$ in the No-Enforcement game to $13.6 \%$ in the with-enforcement game). In the LIHS condition, the accuracy-violation rates decreased by $14.5 \%$ ( $S D=24.0$; from $36.9 \%$ in the No-Enforcement game to $22.3 \%$ in the withenforcement game). Again, the Shapiro-Wilk test rejected the assumption of normality for the accuracy-violation rates $(\mathrm{p}<.001)$. A Mann-Whitney U-test indicated that the reduction of accuracy-violation rates in the HILS condition ( $\operatorname{mdn}=18.1 \%$, range $[-34,109]$ ) was greater than in the LIHS condition ( $\mathrm{mdn}=10 \%$, range $[-29,86]), \mathrm{U}=5291, p=.038$, one-tailed test, effect size $r=0.128$. This finding thus further supports our hypothesis that even when the severity of the fine is provided explicitly as means of deterrence, enforcement with a high probability of inspection and low fines is still more effective in reducing violation behavior than enforcement with severe fines but a low probability of inspection.

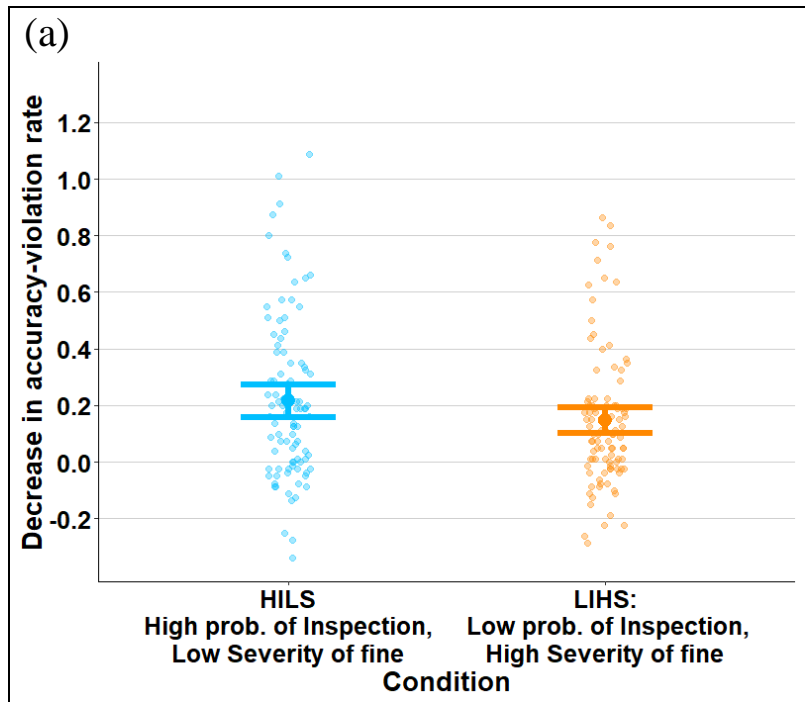

(b)

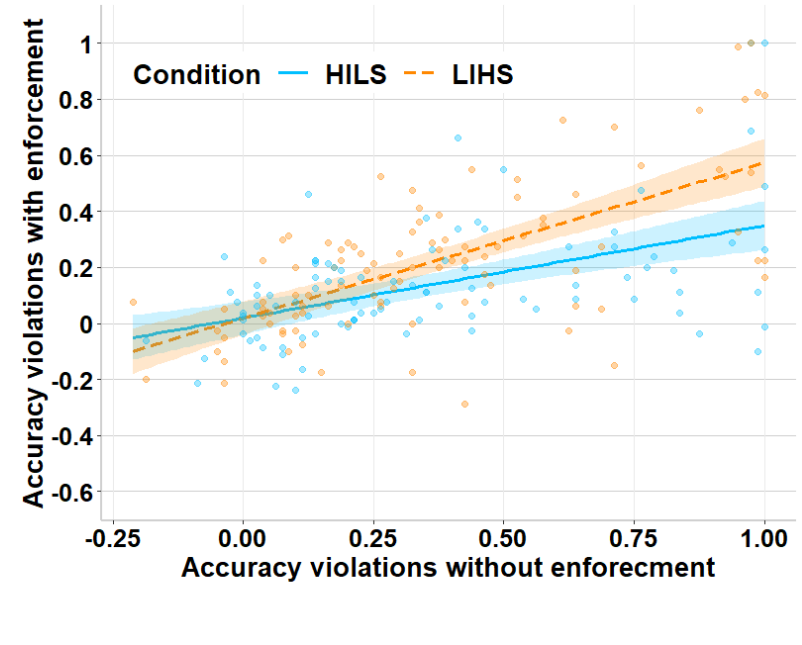

Figure 3. Results of Study 2. Small dots depict individual participants. (a) Decrease in accuracyviolation rates from baseline to the enforcement block by condition. Error bars represent the $95 \%$ bootstrapped CI for the mean. (b) Regression lines of the accuracy-violation rates in the enforcement blocks on the accuracy-violation rates in the No-Enforcement block, by condition. Shading around the lines are the $95 \%$ CIs.

Again, we found no evidence that the frequent inspections caused participants in the HILS condition to make fewer innocent mistakes than in the LIHS condition. A Welch two sample t-test revealed no difference between the two groups $(M=1.8 \%, S D=7.2$ vs. $M=1.3 \%, S D=9.3$ in the HILS and LIHS conditions respectively), $t(182.5)=-0.36, p=0.72$. Thus, the increased 
effectivity of the HILS over the LIHS enforcement could not be attributed to feedback improving perceptual accuracy.

Testing the secondary hypothesis. A regression analysis using the continuous accuracyviolation rates in the No-Enforcement game indicated that the difference between the two enforcement conditions was more pronounced when the accuracy-violation rates in the NoEnforcement game were higher (Figure 3b). The interaction with condition was significant, $F(1,188)=6.49, p=0.012, b=0.22,95 \%$ CI $[0.05,0.40]$, partial $f^{2}=0.03$. Specifically, the simple slopes of the accuracy-violation rate with enforcement on the accuracy-violation without enforcement were $0.33,95 \%$ CI $[0.21,0.45]$ for HILS and $0.55,95 \%$ CI $[0.43,0.68]$ for LIHS.

\section{Discussion}

What is the best way to reduce violations? The high cost of close monitoring and severe sanctions (e.g. more officers, negative mass reactance) render full enforcement impractical. Instead, two common enforcement policies trade off the probability of inspection with the severity of punishment. Our findings suggest that in repeated settings, people are more sensitive to the probability of inspection than to the severity of the fine. In all three studies, the High Inspection Low Severity (HILS) policy was more effective at reducing violation rates than the Low Inspection High Severity (LIHS) policy, despite the equal EV. This finding was independent of potential improvement in perceptual skill, and held even when we increased deterrence by providing information about the magnitude of the fine in advance. Furthermore, the advantage of the HILS policy was more pronounced among participants who tended to commit more violations at baseline.

These findings are consistent with the decisions from experience hypothesis and specifically with the phenomenon of underweighting of rare events (e.g., Newell \& Rakow, 2007). According to this phenomenon, in repeated settings, rare severe punishments are underweighted, causing deterrence to lose its initial effect. Underweighting of rare events is often argued to be the outcome of a decision mechanism that relies on inference derived from small samples of past experiences (e.g., Plonsky et al., 2015). On each trial, decision makers only consider a small sample of previous experiences with each of the options, and choose the option with the higher sample mean. Since small samples usually include frequent outcomes and rare outcomes are under-represented, repeated choices are driven by the more frequent outcomes. In the current context, this mechanism explains why committing violations was more attractive when violations were rewarded most of the time (in LIHS) and less attractive when violations incurred a 
fine most of the time (in HILS). Interestingly, our findings are also consistent with empirical studies showing that crime rates are more strongly correlated with enforcement prevalence than with criminal sanctions (Chalfin \& McCrary, 2017). Our controlled experimental setting suggests a causal link between enforcement prevalence and violation rates for a general (non-criminal) population.

Our results run counter to the economic hypothesis, which posits that severe punishments are more effective than high monitoring in reducing violations in the general population (Becker, 1968; Anderson \& Stafford, 2003; Block \& Gerety, 1995; Friesen, 2012). One plausible reason for this discrepancy is that experimental studies supporting the economic prediction tend to employ an explicit descriptive setting with very few opportunities for violation. The current studies employed an arguably more ecologically valid setting, where implicit opportunities to violate were repeated and the probability of inspection was not described a-priori. Hence, the difference could be the result of a description-experience gap in decisions to violate rules, similar to the gap found in risky choice (Hertwig \& Erev, 2009).

Our main result also diverges from the moral-self maintenance hypothesis, which emphasizes internal costs and implies that external enforcement has limited effectiveness. Previous findings showing the insensitivity to the probability of getting caught (e.g., Mazar et al., 2008; Gamliel \& Peer, 2013) could be attributed to the focus on short-term effects. The current findings reveal a strong sensitivity to the external probability of getting caught and thus demonstrate the importance of investigating long-term learning effects in behavioral ethics. However, the observation that under no external enforcement, most of the participants did not maximize profits is consistent with the suggested role of internal cost as a gatekeeper of morality.

From a practical standpoint, our findings suggest that policy-makers should prioritize high inspection rate over severe punishment. While increasing the magnitude of fines is often perceived as less costly (Becker, 1968), large fines could result in a perception of unfairness and consequently reduce the probability of detection (Feess et al., 2018; Polinsky \& Shavell, 2000), which according to our results, is the key ingredient. Thus, while many regulators in the current Covid-19 pandemic have publicly called to increase the magnitude of fines, our findings clearly suggest that "gentle rule enforcement" that includes small punishments with high probability (Erev et al., 2010; Schurr et al., 2014; Erev et al., 2020; Roth et al., 2020) would be more effective in reducing violation rates, especially for high offenders, the target population of any enforcement policy. 


\section{References:}

Abeler, J., Becker, A., \& Falk, A. (2014). Representative evidence on lying costs. Journal of Public Economics, 113, 96-104.

Anderson, L. R., \& Stafford, S. L. (2003). Punishment in a regulatory setting: experimental evidence from the VCM. Journal of Regulatory Economics, 24(1), 91-110.

Ayal, S., \& Gino, F. (2011). Honest rationales for dishonest behavior. In M. Mikulincer \& P. R. Shaver (Eds.), The social psychology of morality: Exploring the causes of good and evil, pp. 149166. Washington, DC: American Psychological Association.

Barkan, R., Ayal, S., \& Ariely, D. (2015). Ethical dissonance, justifications, and moral behavior. Current Opinion in Psychology, 6(DEC), 157-161.

Becker, G. S. (1968). Crime and punishment: An economic approach. In The economic dimensions of crime (pp. 13-68). Palgrave Macmillan, London.

Block, M. K., \& Gerety, V. E. (1995). Some experimental evidence on differences between student and prisoner reactions to monetary penalties and risk. The Journal of Legal Studies, 24(1), 123-138.

Engel, C., \& Nagin, D. (2015). Who is afraid of the stick? Experimentally testing the deterrent effect of sanction certainty. Review of Behavioral Economics, 2(4), 405-434

Erev, I., Ingram, P., Raz, O., \& Shany, D. (2010). Continuous punishment and the potential of gentle rule enforcement. Behavioural Processes, 84(1), 366-371.

Erev, I., Plonsky, O., \& Roth, Y. (2020). Complacency, panic, and the value of gentle rule enforcement in addressing pandemics. Nature Human Behaviour, 4(11), 1095-1097.

Feess, E., Schildberg-Hörisch, H., Schramm, M., \& Wohlschlegel, A. (2018). The impact of fine size and uncertainty on punishment and deterrence: Theory and evidence from the laboratory. Journal of Economic Behavior \& Organization, 149, 58-73.

Feldman, Y. (2018). The law of good people: Challenging states' ability to regulate human behavior. Cambridge University Press.

Fischbacher, U., \& Föllmi-Heusi, F. (2013). Lies in disguise - an experimental study on cheating. Journal of the European Economic Association, 11(3), 525-547.

Friesen, L. (2012). Certainty of punishment versus severity of punishment: An experimental investigation. Southern Economic Journal, 79(2), 399-421.

Gamliel, E., \& Peer, E. (2013). Explicit risk of getting caught does not affect unethical behavior. Journal of Applied Social Psychology, 43(6), 1281-1288.

Gerlach, P., Teodorescu, K., \& Hertwig, R. (2019). The truth about lies: A meta-analysis on dishonest behavior. Psychological bulletin, 145(1), 1.

Gino, F., Ayal, S., \& Ariely, D. (2013). Self-serving altruism? The lure of unethical actions that benefit others. Journal of economic behavior \& organization, 93, 285-292.

Gneezy, U., Imas, A., \& Madarász, K. (2014). Conscience accounting: Emotion dynamics and social behavior. Management Science, 60(11), 2645-2658.

Gneezy, U., Rockenbach, B., \& Serra-Garcia, M. (2013). Measuring lying aversion. Journal of Economic Behavior \& Organization, 93, 293-300. 
Hilbig, B. E., \& Zettler, I. (2015). When the cat's away, some mice will play: A basic trait account of dishonest behavior. Journal of Research in Personality, 57, 72-88.

Hochman, G., Glöckner, A., Fiedler, S., \& Ayal, S. (2016). "I can see it in your eyes": Biased Processing and Increased Arousal in Dishonest Responses. Journal of Behavioral Decision Making, 29(2-3), 322-335.

Jessup, R. K., Bishara, A. J., \& Busemeyer, J. R. (2008). Feedback produces divergence from prospect theory in descriptive choice. Psychological Science, 19(10), 1015-1022.

Kajackaite, A., \& Gneezy, U. (2017). Incentives and cheating. Games and Economic Behavior, 102, 433-444.

Marchiori, D., Di Guida, S., \& Erev, I. (2015). Noisy retrieval models of over-and undersensitivity to rare events. Decision, 2(2), 82.

Mitchell Polinsky, A., \& Shavell, S. (2000). The fairness of sanctions: some implications for optimal enforcement policy. American Law and Economics Review, 2(2), 223-237.

Newell, B. R., \& Rakow, T. (2007). The role of experience in decisions from description. Psychonomic Bulletin \& Review, 14(6), 1133-1139.

Plonsky, O., \& Teodorescu, K. (2020). The influence of biased exposure to forgone outcomes. Journal of Behavioral Decision Making, 33(3), 393-407.

Plonsky, O., Teodorescu, K., \& Erev, I. (2015). Reliance on small samples, the wavy recency effect, and similarity-based learning. Psychological review, 122(4), 621.

Roth Y., Plonsky O., Shalev E. \& Erev I. (2020) On the Value of Alert Systems and Gentle Rule Enforcement in Addressing Pandemics. Frontiers in Psychology, 11:577743.

Sachdeva, S., Iliev, R., \& Medin, D. L. (2009). Sinning saints and saintly sinners: The paradox of moral self-regulation. Psychological science, 20(4), 523-528.

Shalvi, S., Gino, F., Barkan, R., \& Ayal, S. (2015). Self-serving justifications: Doing wrong and feeling moral. Current Directions in Psychological Science, 24(2), 125-130.

Schurr, A., Rodensky, D., \& Erev, I. (2014). The effect of unpleasant experiences on evaluation and behavior. Journal of Economic Behavior \& Organization, 106, 1-9.

Teodorescu, K., \& Erev, I. (2014a). On the decision to explore new alternatives: The coexistence of under-and over-exploration. Journal of Behavioral Decision Making, 27(2), 109-123.

Teodorescu, K., \& Erev, I. (2014b). Learned helplessness and learned prevalence: Exploring the causal relations among perceived controllability, reward prevalence, and exploration.

Psychological science, 25(10), 1861-1869. 